\title{
Fungicide Resistance in Botrytis fragariae and Species Prevalence in the Mid-Atlantic United States
}

Madeline E. Dowling, Meng-Jun Hu, and Guido Schnabel, ${ }^{\dagger}$ Department of Plant and Environmental Sciences, Clemson University, Clemson, SC 29634

\begin{abstract}
Botrytis fragariae was recently described causing gray mold of strawberry in Germany and the United States. The goal of the present study was to determine its prevalence, distribution, and sensitivity to fungicides in strawberry fields of five states. In total, 188 Botrytis isolates were obtained from flowers and fruit collected from the states of Maryland $(n=35)$, Virginia $(n=38)$, North Carolina $(n=46)$, South Carolina $(n=41)$, and Georgia $(n=28)$. Only 13 of these were fruit samples and came from South Carolina $(n=5)$ and Georgia $(n=8)$. B. fragariae made up $35.1 \%$ of the entire collection, and composed close to half of the Botrytis population in North Carolina (43.4\%), South Carolina (61.0\%), and Georgia (42.9\%). One isolate of $B$. mali was also found, and the rest of the isolates were $B$. cinerea (sensu lato). $B$. fragariae and $B$. cinerea were found coexisting in 11 fields, while other field samples consisted of only B. fragariae $(n=3)$ or only B. cinerea $(n=10)$ isolates. $B$. fragariae

isolates with resistance to one or more fungicides were found, and resistance profiles differed from those of $B$. cinerea, in that no resistance to cyprodinil (FRAC 8) or boscalid and other FRAC 7 botryticides was detected. We detected $B$. fragariae resistance to the active ingredients thiophanate-methyl, iprodione, fludioxonil, and fenhexamid. We also detected $B$. fragariae isolates with resistance to up to four chemical classes of fungicides, though most isolates were resistant to one or two chemical classes. In conclusion, isolates of the newly detected species $B$. fragariae were commonly found on strawberry flowers in the Mid-Atlantic United States, and have developed resistance to many of the most commonly used botryticides. Though the relevance of this species to pre- and postharvest fruit infections is unknown, fludioxonil applications may give this species a competitive advantage over B. cinerea. Controlling this fungus with FRAC 7 fungicides may be an effective way of limiting its spread in strawberry fields.
\end{abstract}

Gray mold is one of the most devastating diseases on strawberry, and is most commonly caused by the pathogen Botrytis cinerea (Fillinger and Elad 2015; Maas 1998). For many years, B. cinerea has been viewed as the only major pathogen causing gray mold on strawberry in the United States, because other species causing this disease have limited prevalence (Amiri et al. 2016; Dowling and Schnabel 2017b; Li et al. 2012a; Maas 1998). However, in Europe, 38 isolates of a newly described species, B. fragariae, were recovered from strawberry vegetative tissue and fruit in fields of four different German states, making up $2.5 \%$ of the collection population (Rupp et al. 2017). Though this percentage may seem insignificant compared with $B$. cinerea frequencies, it indicates that $B$. fragariae is common and geographically widespread in comparison with other minor $B o$ trytis spp. previously detected on strawberry that were found at low frequencies in few fields (Amiri et al. 2016; Dowling and Schnabel 2017b; Li et al. 2012a).

During a regional fungicide resistance monitoring program, 39 isolates of $B$. fragariae were detected in the states of Ohio, North Carolina, and South Carolina around the same time that isolates of the same species were detected in Europe (Dowling et al. 2017a; Rupp et al. 2017). The isolates from the United States were distinct from $B$. cinerea isolates in their cultural characteristics, sensitivity to polyoxin $\mathrm{D}$, and inoculum source. However, no studies have yet been performed to determine the prevalence of this species in the United States. Another aspect of B. fragariae that separates it from

${ }^{\dagger}$ Corresponding author: G. Schnabel; E-mail: schnabe@clemson.edu

Funding: This project was supported by Technical Contribution Number 6559 of the Clemson University Experiment Station and by the United States Department of Agriculture National Institute of Food and Agriculture, under project number 2014-51181-22377 and SC-1700501.

*The $\boldsymbol{e}$-Xtra logo stands for "electronic extra" and indicates that two supplementary figures are published online.

Accepted for publication 2 December 2017.

() 2018 The American Phytopathological Society other Botrytis spp. causing gray mold on strawberry is its unique fungicide resistance patterns (Rupp et al. 2017). B. fragariae in Germany had resistance to up to five chemical classes of fungicides, and individual isolates exhibited multiple chemical class resistance (CCR) to up to four chemical classes. In some cases, B. fragariae exhibited greater resistance frequencies than B. cinerea (Rupp et al. 2017).

The goal of this study was to (i) determine the prevalence of B. fragariae in strawberry fields of the Mid-Atlantic United States, (ii) determine whether this species is found in mixture or in single species populations, and (iii) determine resistance to commonly used chemical classes of fungicides.

\section{Materials and Methods}

Sample collection and isolation. Symptomatic strawberry blossoms and fruit were obtained from 24 commercial strawberry fields in Maryland (4 fields), Virginia (5 fields), North Carolina (6 fields), South Carolina (6 fields), and Georgia ( 3 fields) during bloom from February to March 2016 (Table 1). Fruit samples were obtained in February from one location in Georgia and one location in South Carolina that used high tunnel or greenhouse production for early harvesting. Three fields were from the same geographic location; therefore, isolates from these fields were grouped together to form a total of 21 geographically distinct locations (Fig. 1). Tissue samples were surface sterilized and kept at $100 \%$ relative humidity for 3 days, as described previously, until the fungus produced spores (Fernández-Ortuño et al. 2014). Single-spore isolations were performed as described previously (Dowling et al. 2016a) to obtain 188 Botrytis isolates from 175 blossoms and 13 fruit. No location was represented by more than 10 single-spore isolates, with an average of 7.8 isolates/field and no fewer than 5 isolates/field (Table 1). We obtained fungicide spray records for some fields (Table 1), which indicated that, after planting but prior to bloom, many commercial growers followed spray guide recommendations and exclusively used multisite fungicides (Fungicide Resistance Action Committee [FRAC] M) (Brannen et al. 2017).

PCR-based isolate identification. Isolates were identified to the species level using a previously published polymerase chain reaction (PCR) assay (Dowling et al. 2017a). Reactions consisted of a small, barely visible amount of fungal hyphae for DNA template (obtained from fungal cultures grown on potato dextrose agar for 1 week), $1 \mu l$ 
of each primer (20 $\mu \mathrm{M}$ concentration), hot start Taq polymerase PCR mixture (Bioneer, Alameda, CA), and $16 \mu$ l of DNase-free sterile water for a total reaction volume of $20 \mu$ l. PCR products were analyzed by gel electrophoresis on $1.5 \%$ agarose gels.

Fungicide resistance screening. We screened B. fragariae isolates for resistance to the most commonly used FRAC modes of action used to control Botrytis spp. Isolate sensitivities to cyprodinil (anilinopyrimidine; FRAC 9), boscalid (succinate dehydrogenase inhibitor [SDHI]; FRAC 7), penthiopyrad (FRAC 7), fluopyram (FRAC 7), pydiflumetofen (FRAC 7), isofetamid (FRAC 7), iprodione (dicarboxamide; FRAC 2), fenhexamid (hydroxyanilide; FRAC 17), fludioxonil (phenylpyrrole; FRAC 12), thiophanate-methyl (methyl benzimidazole carbamate; FRAC 1), and polyoxin-D (chitin synthase inhibitor; FRAC 19) were determined based on visual assessment of radial mycelial growth on discriminatory doses of fungicide. This testing was performed as part of a regional fungicide resistance monitoring study using media and methods previously validated by comparison with spore germination analysis (Fernández-Ortuño et al. 2014). In this study, we replaced pyraclostrobin with polyoxin D at $5 \mu \mathrm{g} / \mathrm{ml}$ (Dowling et al. 2016b), because pyraclostrobin is technically not a botryticide and only provides suppressive activity against Botrytis spp. according to the Cabrio EG (BASF, Research Triangle Park, NC) product label. Formulations, FRAC codes, media, and discriminatory doses of all fungicides used are shown in Table 2. Fungicides were chosen to represent singlesite modes of action currently registered for gray mold control in the United States. SDHI fungicides (FRAC 7) were represented with five active ingredients of different chemistry (pyridine-carboxamide, pyrazole-4-carboxamide, pyridinyl-ethyl-benzamide, phenyl-oxo-ethyl thiophene amide, and N-methoxy-[phenyl-ethyl]-pyrazole-carboxamide) due to incomplete cross resistance among FRAC 7 chemistries (Stammler et al. 2015). Isolates were cultured on various media amended with discriminatory doses of fungicides (Table 2) and grouped into the categories sensitive (S), reduced sensitive (RS), moderately resistant (MR), and resistant (R) to each fungicide based on visual mycelial growth assessment (Fernández-Ortuño et al. 2014). After 4 days of incubation in the dark at $22^{\circ} \mathrm{C}$, mycelial growth was assessed. Isolates were designated as $\mathrm{S}$ if no growth was observed, RS if growth was less than $3 \mathrm{~mm}$, MR if growth was between 3 and $7.5 \mathrm{~mm}, \mathrm{R}$ if growth was greater than $7.5 \mathrm{~mm}$ in a 15 -mm-diameter well. Our RS category for the fungicides cyprodinil, iprodione, fenhexamid, and fludioxonil corresponds to the low resistance category described by FernándezOrtuño et al. (2014). For polyoxin D, all isolates able to grow at

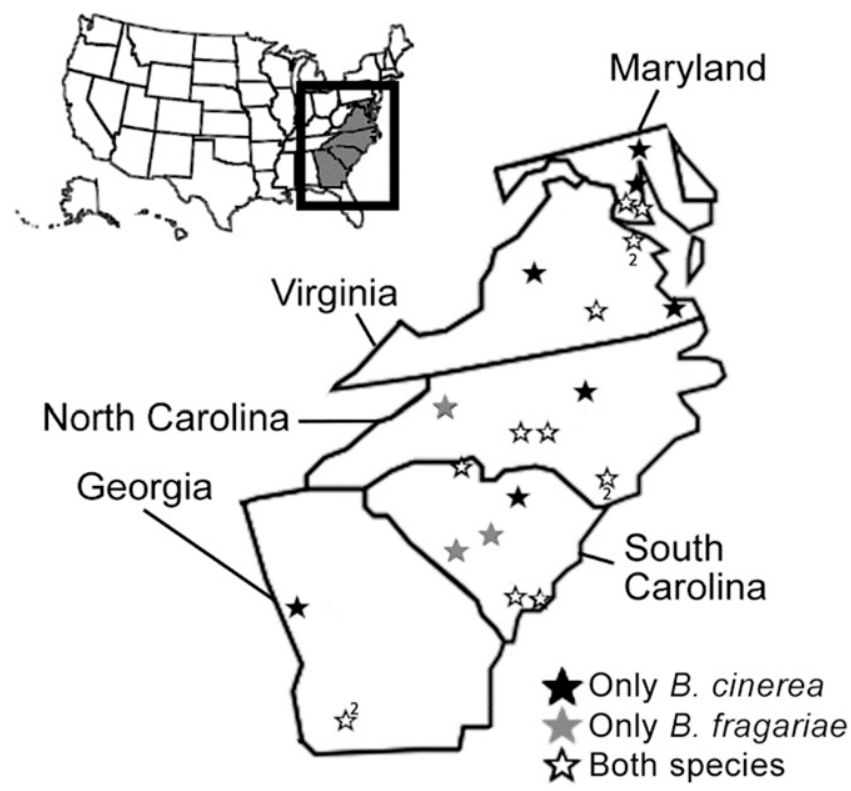

Fig. 1. Map of collection locations and species found at each town or city within the five states used in this study. An average of 7.8 isolates was sampled from each of 24 fields and 21 geographically distinct locations. Three of these locations contained more than one field. Locations containing more than one field are marked with numbers denoting how many fields are represented by that location.

Table 1. Overview of isolates collected from each location in 2016, showing the frequency of each species and the distribution of species within fields

\begin{tabular}{|c|c|c|c|c|c|c|c|c|c|}
\hline State $^{\mathbf{a}}$ & Loc $^{\mathbf{b}}$ & Field $^{c}$ & Species detected $^{\mathrm{d}}$ & Isolates $(n)$ & $B c(n)$ & $B f(n)$ & Sampling month & Sample tissue & FRAC codes applied \\
\hline $\mathrm{MD}$ & 1 & 1 & $\mathrm{Bc}$ & 7 & 7 & 0 & March & Flower & Unknown \\
\hline$M D$ & 2 & 2 & $B c \& B f$ & 8 & 6 & 2 & March & Flower & M4 \\
\hline$M D$ & 3 & 3 & $B c$ & 10 & 10 & 0 & March & Flower & M4 \\
\hline$M D$ & 4 & 4 & $B c \& B f$ & 10 & 7 & 3 & March & Flower & M3, M4 \\
\hline VA & 5 & 5 & $\mathrm{Bc} \& \mathrm{Bf}$ & 5 & 4 & 1 & February & Flower & Unknown \\
\hline$V A$ & 6 & 6 & $B c$ & 10 & 10 & 0 & March & Flower & M4 \\
\hline$V A$ & 6 & 7 & $B c \& B f$ & 10 & 7 & 3 & March & Flower & M4 \\
\hline VA & 7 & 8 & $\mathrm{Bc}$ & 7 & 7 & 0 & March & Flower & Unknown \\
\hline VA & 8 & 9 & $\mathrm{Bc}$ & 6 & 6 & 0 & March & Flower & Unknown \\
\hline $\mathrm{NC}$ & 9 & 10 & $\mathrm{Bc}$ & 5 & 5 & 0 & March & Flower & Unknown \\
\hline NC & 10 & 11 & $\mathbf{B c} \& \mathbf{B f}$ & 10 & 5 & 5 & March & Flower & Unknown \\
\hline$N C$ & 11 & 12 & $B c$ & 9 & 9 & 0 & February & Flower & $7,11, M 4$ \\
\hline$N C$ & 11 & 13 & $B c \& B f$ & 7 & 1 & 6 & February & Flower & $7,11, M 4$ \\
\hline $\mathrm{NC}$ & 12 & 14 & $\mathrm{Bc}$ & 6 & 6 & 0 & February & Flower & Unknown \\
\hline NC & 13 & 15 & $\mathrm{Bf}$ & 9 & 0 & 9 & February & Flower & Unknown \\
\hline $\mathrm{SC}$ & 14 & 16 & $\mathrm{Bf}$ & 9 & 0 & 9 & February & Flower & Unknown \\
\hline $\mathrm{SC}$ & 15 & 17 & $\mathrm{Bc} \& \mathrm{Bf}$ & 5 & 1 & 4 & February & Flower & Unknown \\
\hline SC & 16 & 18 & $\mathrm{Bf}$ & 10 & 0 & 10 & March & Flower & Unknown \\
\hline $\mathrm{SC}$ & 17 & 19 & $\mathrm{Bc}$ & 5 & 5 & 0 & February & Fruit & Unknown \\
\hline $\mathrm{SC}$ & 18 & 20 & $\mathrm{Bc} \& \mathrm{Bf}$ & 6 & 5 & 1 & February & Flower & Unknown \\
\hline $\mathrm{SC}$ & 19 & 21 & $\mathrm{Bc} \& \mathrm{Bf}$ & 6 & 5 & 1 & February & Flower & Unknown \\
\hline GA & 20 & 22 & Bc \& Bf & 9 & 4 & 5 & February & Flower & Unknown \\
\hline GA & 20 & 23 & $\mathrm{Bc} \& \mathrm{Bf}$ & 8 & 1 & 7 & February & Fruit & Unknown \\
\hline GA & 21 & 24 & $\mathrm{Bc}$ & 10 & 10 & 0 & March & Flower & Unknown \\
\hline
\end{tabular}

${ }^{\mathrm{a}}$ Locations with information in bold were included in the smaller pool of isolates to control for sampling and species bias shown in the supplementary figures. Locations in italics provided fungicide application records from planting to bloom in 2016. States: Maryland (MD), Virginia (VA), North Carolina (NC), South Carolina (SC), and Georgia (GA).

${ }^{\mathrm{b}}$ Locations (Loc) are defined as the number of city areas where samples were collected.

${ }^{\mathrm{c}}$ Several locations contained more than one field.

d The designation "Bc" and "Bf" refer to Botrytis cinerea and Botrytis fragariae, respectively.

${ }^{\mathrm{e}} \mathrm{FRAC}$ codes applied after planting but prior to sampling. 
the $5 \mathrm{ppm}$ discriminatory dose were designated RS (Dowling et al. 2016b).

Statistical analysis. To determine whether the distribution of sensitivities to each fungicide was different between the two species B. fragariae and B. cinerea, as it was in Germany, statistical analyses

Table 2. Active ingredients and their concentrations, formulations, and media used to assess sensitivity of Botrytis fragariae and B. cinerea to selected fungicides

\begin{tabular}{llccc}
\hline $\begin{array}{l}\text { Active } \\
\text { ingredient }\end{array}$ & Trade name & $\begin{array}{c}\text { FRAC } \\
\text { code }\end{array}$ & $\begin{array}{c}\text { Concentration } \\
(\boldsymbol{\mu} \mathbf{g} / \mathbf{m l})^{\mathbf{a}}\end{array}$ & Medium $^{\mathbf{b}}$ \\
\hline $\begin{array}{l}\text { Thiophanate- } \\
\text { methyl }\end{array}$ & Topsin-M & 1 & 100 & MEA \\
Iprodione & Rovral 4F & 2 & 10 & MEA \\
Boscalid & Endura & 7 & 75 & YBA \\
Fluopyram & Luna Privilege & 7 & 10 & YBA \\
Penthiopyrad & Fontelis & 7 & 5 & YBA \\
Isofetamid & Kenja 400SC & 7 & 5 & YBA \\
Pydiflumetofen & Adepidyn & 7 & 5 & YBA \\
Cyprodinil & Vangard WG & 9 & 4 & CzA \\
Fludioxonil & Scholar & 12 & 0.5 & MEA \\
Fenhexamid & Elevate 50WDG & 17 & 50 & MEA \\
Polyoxin D & Ph-D WDG & 19 & 5 & MEA \\
\hline
\end{tabular}

a Concentration of active ingredient.

${ }^{b}$ Malt extract agar (MEA), yeast beef agar (YBA), and Czapek-Dox agar (CzA).

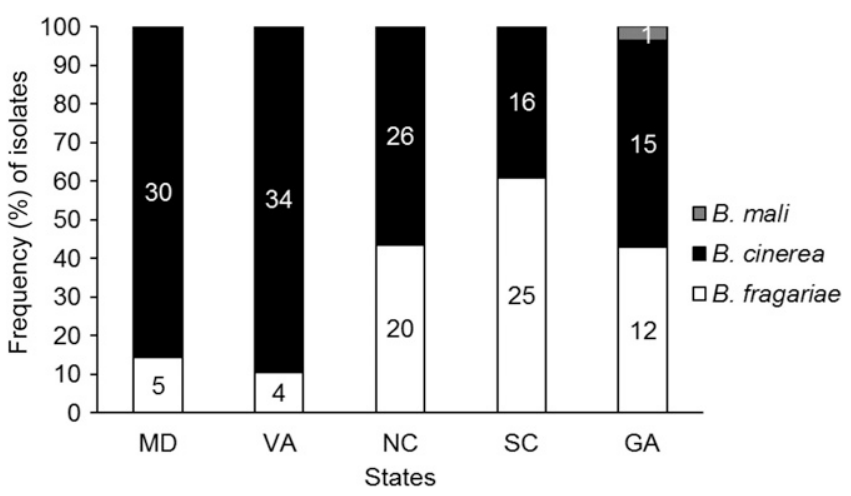

Fig. 2. Prevalence of Botrytis fragariae, $B$. cinerea, and $B$. mali from strawberry flowers and fruit in 24 total fields within the states of Maryland (MD; 4 fields), Virginia (VA; 5 fields), North Carolina (NC; 6 fields), South Carolina (SC; 6 fields), and Georgia (GA; 3 fields) were performed. For cyprodinil, fludioxonil, fenhexamid, and iprodione, $\chi^{2}$ analyses were implemented individually, where the distribution of isolates in all four sensitivity categories (, RS, MR, and R) was compared between $B$. fragariae and $B$. cinerea using four-by-four contingency tables. For thiophanate-methyl, boscalid, fluopyram, penthiopyrad, isofetamid, pydiflumetofen and polyoxin D, Fisher's exact tests were performed, because only two sensitivity categories existed for each of these fungicides. All statistical analyses were performed using JMP Pro 12 (SAS Institute, Inc., Cary, NC) using a significance level of $\alpha=0.05$.

\section{Results}

B. fragariae species prevalence. In total, 188 isolates, mostly from flowers of annual strawberry plants, were identified to the species level using species-specific primers (Dowling et al. 2017a). $B$. cinerea made up $64.4 \%$ of the population, followed by $B$. fragariae (35.1\%) and B. mali (0.5\%). Of the 13 isolates obtained from fruit, 7 were $B$. fragariae and 6 were $B$. cinerea. All B. fragariae isolates from fruit were collected from the same field in Georgia. B. fragariae and B. cinerea were found in all five states (Fig. 2). Commercial strawberry fields in Maryland and Virginia mainly contained $B$. cinerea isolates but approximately equal ratios of $B$. fragariae and $B$. cinerea were found in commercial fields from North Carolina, South Carolina, and Georgia. One isolate of B. mali was found in Georgia (Fig. 2). $B$. fragariae and $B$. cinerea coexisted in 11 of the 24 fields sampled. Fields containing both species were found in each of the five states examined. The average frequency of $B$. cinerea and $B$. fragariae isolates in mixed fields was 55 and $45 \%$, respectively.

Fungicide resistance profiles by species. Sensitivity to 11 active ingredients of eight chemical classes of fungicides was determined for all isolates. B. fragariae resistance to thiophanate-methyl, iprodione, fludioxonil, fenhexamid, and polyoxin-D was detected (Fig. 3). Most notably, and in contrast to $B$. cinerea, $B$. fragariae isolates were not resistant to any of the FRAC 7 fungicides tested (boscalid, penthiopyrad, fluopyram, fluxapyroxad, or pydiflumetofen) or to cyprodinil (Fig. 3). Because no resistance to fluopyram, fluxapyroxad, or pydiflumetofen was detected in either species, these fungicides were not included in Figure 3. Because sample sizes and species frequencies in individual fields varied depending on location, results could potentially be biased due to differing location-specific spray histories. However, a subset of isolates was analyzed from five fields where both species coexisted and each was represented by more than one isolate and, where three of the fields were known to have been only sprayed with nonselective, FRAC M fungicides between planting and bloom (Table 1; Supplementary Figs. S1 and S2). Results from this subset agreed with the complete dataset, indicating that results were not affected by fungicide sprays between planting and sampling. There

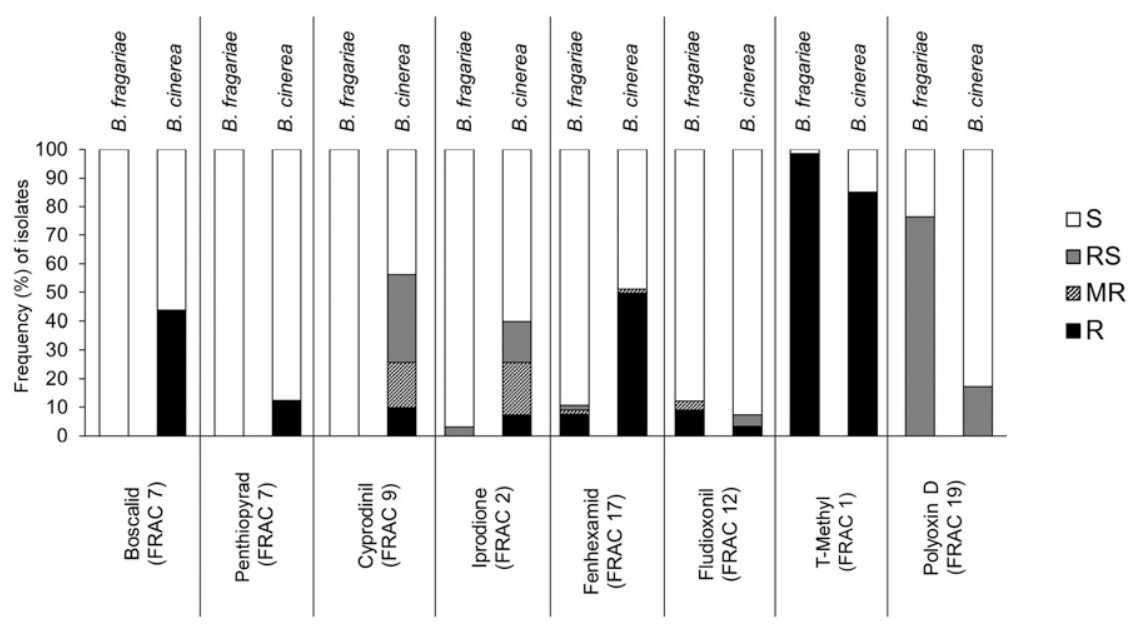

Fig. 3. Fungicide resistance profiles of isolates in our collection displaying resistance (R), moderate resistance (MR), reduced sensitivity (RS), and sensitivity (S) to fungicides from eight chemical classes. Thiophanate-methyl, boscalid, and penthiopyrad have only $\mathrm{R}$ or $\mathrm{S}$ categories because no quantitative resistance to these fungicides has been detected in Botrytis spp. on strawberry. Because no resistance to the FRAC 7 fungicides fluopyram, isofetamid, or pydiflumetofen was detected in either species, they were not included in this figure. 
were more $B$. fragariae than $B$. cinerea isolates with $\mathrm{R}$ to fludioxonil $(P=0.0149)$ and $\mathrm{RS}$ to polyoxin $\mathrm{D}(P=0.0015)$. $B$. fragariae isolates with R to thiophanate-methyl and RS to polyoxin D were found in every state, and fenhexamid resistance was observed in four of the five states. However, B. fragariae isolates resistant to iprodione and fludioxonil were less widespread and found in two and three states, respectively. However, in the five fields where fludioxonil resistance was present and both species coexisted, fludioxonil resistance frequencies were significantly greater for $B$. fragariae isolates than $B$. cinerea isolates $(P<0.0001)$, even though $B$. fragariae was underrepresented

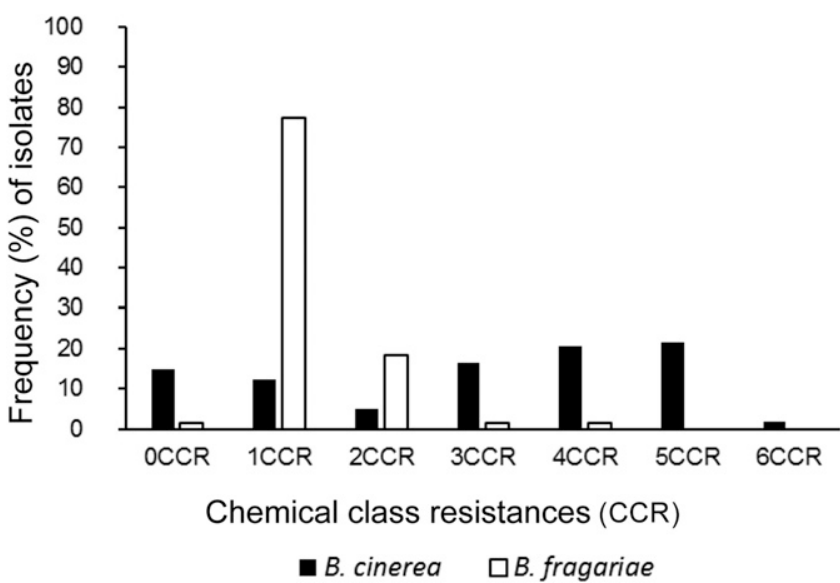

Fig. 4. Frequency of multiple chemical class resistances (CCR) in Botrytis cinerea and $B$. fragariae isolates. The FRAC 11 fungicide pyraclostrobin was not included in this study; thus, the CCR categories are not identical to those of our previous work ( $\mathrm{Hu}$ et al. 2016a; Rupp et al. 2017).

Table 3. Most frequent phenotypes associated with different categories of chemical class resistance (CCR) for Botrytis fragariae and B. cinerea in our collection

\begin{tabular}{|c|c|c|c|c|c|c|c|c|}
\hline \multicolumn{2}{|c|}{ Number of } & \multicolumn{6}{|c|}{ Fungicide $^{\mathbf{a}}$} & \multirow[b]{2}{*}{$\mathrm{CCR}$} \\
\hline B. fragariae & B. cinerea & Tm & $\mathbf{F e}$ & $\mathrm{Cy}$ & Bo & Ip & Fl & \\
\hline 1 & 18 & $S$ & $\mathrm{~S}$ & $S$ & $\mathrm{~S}$ & $S$ & $\mathrm{~S}$ & OCCR \\
\hline 51 & 23 & $\mathbf{R}$ & $\mathrm{S}$ & $\mathrm{S}$ & $\mathrm{S}$ & $\mathrm{S}$ & $\mathrm{S}$ & $1 \mathrm{CCR}$ \\
\hline 6 & 0 & $\mathbf{R}$ & $\mathrm{S}$ & $\mathrm{S}$ & $\mathrm{S}$ & $\mathrm{S}$ & $\mathbf{R}$ & $2 \mathrm{CCR}$ \\
\hline 6 & 3 & $\mathbf{R}$ & $\mathbf{R}$ & $\mathrm{S}$ & $\mathrm{S}$ & $\mathrm{S}$ & $\mathrm{S}$ & $2 \mathrm{CCR}$ \\
\hline 0 & 8 & $\mathbf{R}$ & $\mathbf{R}$ & $\mathbf{R}$ & $\mathrm{S}$ & $\mathrm{S}$ & $\mathrm{S}$ & $3 \mathrm{CCR}$ \\
\hline 1 & 0 & $\mathbf{R}$ & $\mathrm{S}$ & $\mathrm{S}$ & $\mathrm{S}$ & $\mathbf{R}$ & $\mathbf{R}$ & $3 \mathrm{CCR}$ \\
\hline 0 & 11 & $\mathbf{R}$ & $\mathbf{R}$ & $\mathbf{R}$ & $\mathbf{R}$ & $\mathrm{S}$ & $\mathrm{S}$ & $4 \mathrm{CCR}$ \\
\hline 1 & 0 & $\mathbf{R}$ & $\mathbf{R}$ & S & $\mathrm{S}$ & $\mathbf{R}$ & $\mathbf{R}$ & $4 \mathrm{CCR}$ \\
\hline 0 & 24 & $\mathbf{R}$ & $\mathbf{R}$ & $\mathbf{R}$ & $\mathbf{R}$ & $\mathbf{R}$ & $\mathrm{S}$ & $5 \mathrm{CCR}$ \\
\hline 0 & 3 & $\mathbf{R}$ & $\mathbf{R}$ & $\mathbf{R}$ & $\mathbf{R}$ & $\mathbf{R}$ & $\mathbf{R}$ & $6 \mathrm{CCR}$ \\
\hline
\end{tabular}

a Sensitive (S), reduced sensitive (RS), moderately resistant (MR), and resistant $(\mathrm{R})$. Fungicide names are abbreviated as follows: $\mathrm{Tm}=$ thiophanatemethyl, $\mathrm{Fe}=$ fenhexamid, $\mathrm{Cy}=$ cyprodinil, $\mathrm{Bo}=$ boscalid, $\mathrm{Ip}=$ iprodione, $\mathrm{Fl}=$ fludioxonil. in each population. This information is preliminary, due to small sample sizes, but the data are relevant because this phenomenon was observed in five separate fields in three states.

Multiple fungicide resistance. We also investigated the frequency of isolates from each species with multiple CCR using the same two pools of isolates described above (Fig. 4). B. fragariae isolates exhibited fewer multiple CCR compared with $B$. cinerea and most isolates were in the 1CCR and 2CCR categories. Table 3 provides the most common phenotypes of each CCR level. Single isolates of $B$. fragariae were present in each of the 3CCR and 4CCR categories and none were in the $5 \mathrm{CCR}$ or $6 \mathrm{CCR}$ categories. In contrast, more than $60 \%$ of the $B$. cinerea isolates made up 3CCR to 6CCR categories, with three isolates being resistant to six chemical classes of fungicides (6CCR). In the smaller pool of isolates, results were again similar to the overall isolate pool (Fig. 4). Polyoxin D was not included in this analysis because field relevance of RS to polyoxin D has not yet been determined and, therefore, we do not yet consider isolates defined as RS in this study to be "resistant."

\section{Discussion}

B. fragariae was first documented in the United States only recently (Rupp et al. 2017) (Table 4) and, subsequently, differences in polyoxin D sensitivity between this species and $B$. cinerea were reported. Analysis of isolates from various plant sources indicated that inoculum may not derive from nurseries and that $B$. fragariae may prefer strawberry flowers over green or mature fruit (Dowling et al. 2017a). In the current study, we further characterize $B$. fragariae by examining its prevalence across five Mid-Atlantic states, documenting resistance to multiple fungicides, and comparing fungicide resistance profiles with those of $B$. cinerea.

Though only 71 total $B$. fragariae isolates were detected in this study, these isolates made up $35.1 \%$ of our collection population and were present in all five states sampled. The geographic distribution and high prevalence of $B$. fragariae in our collection indicates that the species is common on strawberry flowers in mid-Atlantic states. Most of the fields sampled in this study contained both $B$. cinerea and $B$. fragariae, indicating that they often live in syntopy, despite the previously reported lower fitness of $B$. fragariae (Rupp et al. 2017). Other factors such as fungicide resistance, selection advantage, or host tissue preference may play a role in the interaction that allows $B$. fragariae to coexist with $B$. cinerea. A similar syntopic interaction has been detected between the species $B$. pseudocinerea and $B$. cinerea on strawberry and grape in Europe. Because $B$. pseudocinerea is widely distributed in Europe and has been detected in California on blueberry (Saito et al. 2014), we tested isolates with the fungicide resistance profile typical of B. pseudocinerea using $G 3 P D H$ sequences but did not find this species in our collection (data not shown).

In the current study, B. cinerea and B. fragariae isolates differed in fungicide resistance profiles. In our collection, $B$. fragariae isolates were more likely to have RS to polyoxin $\mathrm{D}$, agreeing with our previous study that showed a significantly larger frequency of $B$. fragariae isolates RS to polyoxin D than B. cinerea isolates (Dowling et al. 2017a). B. fragariae fenhexamid resistance frequencies were lower than those of $B$. cinerea, but resistant isolates were detected in our collection, though they were not observed in Germany (Rupp et al. 2017).

Table 4. Frequency and distribution of Botrytis spp. known to infect strawberry in the United States

\begin{tabular}{|c|c|c|c|c|}
\hline Species & Frequency & States $^{\mathrm{a}}$ & Host plant tissue & Yearb \\
\hline Botrytis cinerea & Common & $\begin{array}{l}\text { All except Nebraska, New Mexico, North Dakota, South Dakota, Utah, } \\
\text { and Vermont }{ }^{c}\end{array}$ & Blossom, leaf, and fruit & 1914 \\
\hline B. fragariae & Common & Ohio, Maryland, Virginia, North Carolina, South Carolina, and Georgia & Blossom, leaf, and fruit & 2015 \\
\hline B. mali & Rare & South Carolina, Maryland & Blossom & 2015 \\
\hline B. caroliniana & Rare & North Carolina & Fruit & 2011 \\
\hline Amphobotrys ricini & Rare & Florida & Blossom, leaf & 2013 \\
\hline
\end{tabular}

${ }^{\text {a }}$ States where each Botrytis spp. was detected on strawberry.

${ }^{b}$ Year detected. Publications describing the first detection of B. cinerea, B. fragariae, B. mali, B. caroliniana, and A. ricini were by Stevens 1914 , Rupp et al. 2017, Dowling and Schnabel 2017b, Li et al. 2012b, and Amiri et al. 2016, respectively.

${ }^{\mathrm{c}}$ Botrytis cinerea is likely present where strawberry production occurs in these states but, to our knowledge, no publications report the fungus in these states. 
We also observed significantly higher frequencies of $B$. fragariae isolates with resistance to fludioxonil than $B$. cinerea isolates collected from the same fields.

Resistance to SDHI (FRAC 7 fungicides) was nonexistent in our collection of $B$. fragariae isolates. This is consistent with the lack of boscalid resistance observed in Germany (Rupp et al. 2017), and indicates that $B$. fragariae may not develop resistance to SDHI fungicides as readily as $B$. cinerea does. This is unusual, because many SDHI resistance mutations have been detected in $B$. cinerea's succinate dehydrogenase (SDH) subunit B gene, including H272Y, H272R, N230I, and P225F (Hahn 2014; Hu et al. 2016b; Veloukas et al. 2013). Resistance to SDHI fungicides may even be conferred by mutations in other SDH subunits, including A85V in SDH subunit C and H132R in SDH subunit D (Fraaije et al. 2012). Possibly, B. fragariae may not tolerate changes in SDH subunits because these subunits may have additional functions in this species that do not allow for much diversity. Or, perhaps, this species has been less exposed to fungicide selection pressure, which would be expected if flowers are, indeed, the main tissue colonized and fruit are not regularly infected (Dowling et al. 2017a). In that case, all applications past the flowering stage would not exhibit selective pressure on the population.

One of the most disturbing characteristics of $B$. cinerea is the ability of single isolates to rapidly develop resistance to multiple chemical classes of fungicides. In a recent study, single isolates were detected with unprecedented resistance to seven major chemical classes of fungicides used to control gray mold (Fernández-Ortuño et al. 2015). This increasing multifungicide resistance is alarming because single-site inhibitor fungicides may become useless in any field where 7CCR isolates are present (Fernández-Ortuño et al. 2015). A recent study shows that these isolates with multiple CCR are likely to be selected by resistance management measures such as fungicide rotations in fields where they exist even at low frequencies (Hu et al. 2016a). Likely, in past studies, B. fragariae isolates were included with $B$. cinerea isolates in studies of multiple CCR. In this study, for the first time in the United States, we analyzed the species separately to clarify the contribution of $B$. fragariae to multiple CCR of Botrytis spp. causing gray mold on strawberry. Almost all $(95.8 \%)$ B. fragariae isolates were $1 \mathrm{CCR}$ (all resistant to thiophanate-methyl) and 2CCR (thiophanate-methyl + fludioxonil or fenhexamid) and very few were 3CCR and 4CCR (Table 3; Fig. 4). This indicates that the species may not have been as exposed to selection by fungicide rotations as B. cinerea. Most German B. fragariae isolates did not exhibit resistance to four, five, and six chemical classes either (Rupp et al. 2017).

Because this study focused on isolates collected from strawberry flowers and not fruit, the economic significance of $B$. fragariae is unclear. Because fruit infections are often initiated in spring during bloom, it is likely that this species will cause at least some preharvest and postharvest fruit rot, similar to B. cinerea (Bulger et al. 1987). Also, some isolates in our collection and previous collections from the United States and Germany originated from fruit, showing that this species is capable of infecting and reproducing on fruit (Dowling et al. 2017a, Rupp et al. 2017). It is unknown whether fruit infections originated in previous blossom infection or from direct infection of wounded or healthy fruit. Lower growth rate and sporulation capacity on fruit and in vitro previously observed in $B$. fragariae isolates indicates that this species may be outcompeted by $B$. cinerea as the season progresses (Dowling et al. 2016b; Rupp et al. 2017). Though B. fragariae's fitness parameters and host tissue preference indicate that it may be of minor economic importance, it is worthy of concern because of its greater frequencies and levels of resistance to fludioxonil and its prevalence in strawberry fields. Only an extensive survey of Botrytis spp. during bloom, preharvest, and postharvest in multiple fields and seasons will fully clarify the importance of this species to commercial strawberry production.

Although B. fragariae's economic importance is undocumented, it has definite practical importance because of its many unique traits. Most certainly, B. fragariae should be distinguished from B. cinerea and other gray mold species when fungicide resistance is of concern. From a disease management perspective, growers struggling with fludioxonil resistance may be battling $B$. fragariae and should consider including a FRAC 7 in their fungicide rotations to decrease the frequency of $B$. fragariae in their fields. Scientifically, it is likely that past research involving gray mold on strawberry unwittingly treated the two species of Botrytis as one, which would have inflated $B$. cinerea fludioxonil resistance frequencies, genetic diversity, and multidrug resistance levels, as well as underestimated $B$. cinerea boscalid resistance frequencies (Fernández-Ortuño et al. 2014; Hu et al. 2016b; Leroch et al. 2013).

In conclusion, we report that, in our collection, B. fragariae was the most prevalent species of Botrytis affecting strawberry flowers in Maryland, Virginia, North Carolina, South Carolina, and Georgia besides $B$. cinerea, and that it is commonly found coexisting with $B$. cinerea in these states. Its fungicide resistance and CCR patterns differ from $B$. cinerea's. In our collection, $B$. fragariae isolates lacked resistance to FRAC 7 fungicides and, relative to $B$. cinerea, more $B$. fragariae isolates were resistant to fludioxonil. Depending on how economically important $B$. fragariae is or will become, gray mold control strategies may have to be adjusted to accommodate this species' characteristics.

\section{Acknowledgments}

We thank K. Bryson and B. Cox for technical assistance.

\section{Literature Cited}

Amiri, A., Onofre, R. B., and Peres, N. A. 2016. First report of gray mold caused by Botryotinia ricini (Amphobotrys ricini) on strawberry in United States. Plant Dis. 100:1007.

Brannen, P. M., Smith, P., Luows, F., Johnson, C., Schnabel, G., Fontenot, K., Pfeiffer, D., Sial, A., and Jennings, K. 2017. 2017 Southeast Regional Strawberry Integrated Pest Management Guide. The Southern Region Small Fruit Consortium. Available from www.smallfruits.org

Bulger, M. A., Ellis, M. A., and Madden, L. V. 1987. Influence of temperature and wetness duration on infection of strawberry flowers by Botrytis cinerea and disease incidence of fruit originating from infected flowers. Phytopathology 77:1225-1230.

Dowling, M. E., Bryson, P. K., Boatwright, H. G., Wilson, J. R., Fan, Z., Everhart S. E., Brannen, P. M., and Schnabel, G. 2016a. Effect of fungicide applications on Monilinia fructicola population diversity and transposon movement. Phytopathology 106:1504-1512.

Dowling, M. E., Hu, M., Schmitz, L. T., Wilson, J. R., and Schnabel, G. 2016b. Characterization of Botrytis cinerea isolates from strawberry with reduced sensitivity to Polyoxin D Zinc Salt. Plant Dis. 100:2057-2061.

Dowling, M. E., Hu, M., and Schnabel, G. 2017a. Identification and characterization of Botrytis fragariae isolates on strawberry in the United States. Plant Dis. 101: $1769-1773$

Dowling, M. E., and Schnabel, G. 2017b. First report of Botrytis mali causing gray mold on strawberry in the United States. Plant Dis. 101:1034.

Fernández-Ortuño, D., Grabke, A., Bryson, P. K., Amiri, A., Peres, N. A., and Schnabel, G. 2014. Fungicide resistance profiles in Botrytis cinerea from strawberry fields of seven southern U.S. States. Plant Dis. 98:825-833.

Fernández-Ortuño, D., Grabke, A., Li, X., and Schnabel, G. 2015. Independent emergence of resistance to seven chemical classes of fungicides in Botrytis cinerea. Phytopathology 105:424-432.

Fillinger, S., and Elad, Y. 2015. Botrytis-The Fungus, the Pathogen and its Management in Agricultural Systems. Springer International Publishing, Cham, Switzerland.

Fraaije, B. A., Bayon, C., Atkins, S., Cools, H. J., Lucas, J. A., and Fraaije, M. W 2012. Risk assessment studies on succinate dehydrogenase inhibitors, the new weapons in the battle to control Septoria leaf blotch in wheat. Mol. Plant Pathol. 13:263-275.

Hahn, M. 2014. The rising threat of fungicide resistance in plant pathogenic fungi: Botrytis as a case study. J. Chem. Biol. 7:133-141.

Hu, M., Cox, K. D., and Schnabel, G. 2016a. Resistance to increasing chemical classes of fungicides by virtue of "Selection by Association" in Botrytis cinerea. Phytopathology 106:1513-1520.

Hu, M., Fernández-Ortuño, D., and Schnabel, G. 2016b. Monitoring resistance to SDHI fungicides in Botrytis cinerea from strawberry fields. Plant Dis. 100: 959-965.

Leroch, M., Plesken, C., Weber, R. W. S., Kauff, F., Scalliet, G., and Hahn, M. 2013. Gray mold populations in German strawberry fields are resistant to multiple fungicides and dominated by a novel clade closely related to Botrytis cinerea. Appl. Environ. Microbiol. 79:159-167.

Li, X., Fernández-Ortuño, D., Chai, W., Wang, F., and Schnabel, G. 2012a. Identification and prevalence of Botrytis spp. from blackberry and strawberry fields of the Carolinas. Plant Dis. 96:1634-1637.

Li, X., Kerrigan, J., Chai, W., and Schnabel, G. 2012b. Botrytis caroliniana, a new species isolated from blackberry in South Carolina. Mycologia 104 650-658. 
Maas, J. 1998. Compendium of Strawberry Diseases, 2nd ed. American Phytopathological Society, St. Paul, MN.

Rupp, S., Plesken, C., Rumsey, S., Dowling, M., Schnabel, G., Weber, R. W. S., and Hahn, M. 2017. Botrytis fragariae, a new species causing gray mold on strawberries, shows high frequencies of specific and efflux-based fungicide resistance. Appl. Environ. Microbiol. 83:e00269-17.

Saito, S., Michailides, T. J., and Xiao, C. L. 2014. First report of Botrytis pseudocinerea causing gray mold on blueberry in North America. Plant Dis. 98:1743.
Stammler, G., Wolf, A., Glaettli, A., and Klappach, K. 2015. Respiration inhibitors: Complex II. Pages 105-117 in: Fungicide Resistance in Plant Pathogens. H. Ishii and D. W. Hollomon, eds. Springer, Tokyo.

Stevens, F. L. 1914. A destructive strawberry disease. Science 39:949-950.

Veloukas, T., Markoglou, A. N., and Karaoglanidis, G. S. 2013. Differential effect of $S d h B$ gene mutations on the sensitivity to SDHI fungicides in Botrytis cinerea. Plant Dis. 97:118-122. 